\title{
Impedimetrische Gassensoren auf Basis von Fe-MFI, Fe-ß und Fe-SAPO-5 zur $\mathrm{NH}_{3^{-}}$oder $\mathrm{NO}_{\mathrm{x}}$-Detektion
}

\author{
Daniela Schönauer-Kamin ${ }^{1}$, Ina Sichert ${ }^{1}$, Daniel Forberg ${ }^{1}$, Michael Schwidder ${ }^{2}$, Ralf Moos ${ }^{1}$ \\ ${ }^{1}$ Lehrstuhl für Funktionsmaterialien, Universität Bayreuth, 95440 Bayreuth \\ ${ }^{2}$ Lehrstuhl für Technische Chemie, Otto-von-Guericke Universität Magdeburg, 39016 Magdeburg
}

\begin{abstract}
Zeolithen wird ein großes Potential als sensitive Schicht für impedimetrische Abgassensoren zugeschrieben. Sie weisen als bekannte Katalysatormaterialien die notwendige Stabilität auf und durch ihre Adsorptionseigenschaften kann eine Vielzahl von Molekülen adsorbiert werden wodurch eine Änderung der elektrischen Eigenschaften denkbar ist. In der vorliegenden Arbeit werden die elektrischen Eigenschaften der SCR-Katalysatoren Fe-MFI, Fe-Beta und des hydrothermal stabileren Fe-SAPO-5 in Abhängigkeit von der Gaszusammensetzung untersucht. Dabei soll die Eignung der Materialien als selektive $\mathrm{NH}_{3}$ oder $\mathrm{NO}_{2}$ Gassensoren quantifiziert werden.
\end{abstract}

\section{Einleitung}

Zeolithe sind in der Literatur als sensitive Schichten für konduktometrische Sensoren bekannt [1, 2]. Die Änderung der ionischen Leitfähigkeit in Abhängigkeit von der Gasadsorption und der Gaskonzentration wird detektiert. Zudem werden Zeolithe aufgrund der hervorragenden katalytischen Aktivität und Selektivität in der Katalyse eingesetzt. Im Bereich der Abgasnachbehandlung zur Minimierung von $\mathrm{NO}_{\mathrm{x}}$-Emissionen hat die selektive katalytische Reduktion (SCR), bei der $\mathrm{NO}_{x}$ mit $\mathrm{NH}_{3}$ selektiv zu $\mathrm{N}_{2}$ und $\mathrm{H}_{2} \mathrm{O}$ umgesetzt wird, zunehmend an Bedeutung gewonnen. Als SCR-Katalysatoren werden neben $\mathrm{V}_{2} \mathrm{O}_{5}-\mathrm{WO}_{3}-\mathrm{TiO}_{2}(\mathrm{VWT})$ verstärkt $\mathrm{Cu}$ - und Fe-haltige Zeolithe (Cu- u. Fe-ZSM5 (MFI)) diskutiert [3]. Der SCR-Mechanismus basiert auf der $\mathrm{NH}_{3}$-Adsorption und den selektiv ablaufenden Umsatzreaktionen unter $\mathrm{NO}_{\mathrm{x}}$-Beteiligung. Neuere Untersuchungen beschäftigen sich mit Fe-haltigen Silico-Alumophosphaten (SAPO) als SCR-Katalysatoren [4], die bei vergleichbarer katalytischer Aktivität eine bessere hydrothermale Stabilität besitzen. Im Folgenden soll untersucht werden, ob sich die SCR-Katalysatoren Fe-MFI, Fe-Beta und Fe-SAPO-5 als sensitive Schichten für impedimetrische Sensoren zur Detektion von $\mathrm{NH}_{3}$ oder $\mathrm{NO}_{\mathrm{x}}$ eignen. VWT wurde in [5] und [6] bereits positiv als Sensorschicht zur $\mathrm{NH}_{3}$ - oder $\mathrm{SO}_{2}$-Detektion charakterisiert

\section{Experimentelles}

In Bild 1 ist der Sensoraufbau impedimetrischer Sensoren zur Charakterisierung der gassensitiven Eigenschaften schematisch gezeigt. Auf einem $\mathrm{Al}_{2} \mathrm{O}_{3}$-Substrat befinden sich GoldInterdigitalelektroden (Au-IDE, 20/20 $\mu \mathrm{m})$ die mit einer siebgedruckten porösen Sensorschicht (Fe-MFI oder $\mathrm{Fe}-\beta$ (Südchemie), Fe-SAPO-5) bedeckt sind. Die Sensorcharakterisierung erfolgt mittels Impedanzspektroskopie $(10 \mathrm{MHz}-1 \mathrm{~Hz}$, Amplitude $1 \mathrm{~V})$ bei einer Sensortemperatur im Bereich von $300{ }^{\circ} \mathrm{C}$ bis $500{ }^{\circ} \mathrm{C}$ in magerem Grundgas $\left(10 \% \mathrm{O}_{2}\right.$, $6,5 \% \mathrm{CO}_{2}, 2,5 \% \mathrm{H}_{2} \mathrm{O}, \mathrm{N}_{2}$ ) unter Zugabe von $\mathrm{NH}_{3}$ und $\mathrm{NO}_{\mathrm{x}}$.

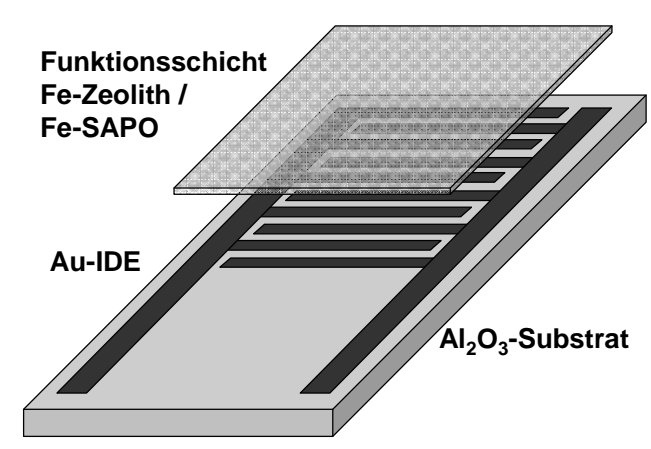

Bild 1 Schema Sensoraufbau

\section{Charakterisierung von Fe-MFI und Fe-Beta als Gassensor}

Im ersten Teil werden die gassensitiven Eigenschaften einer Fe-MFI- und einer Fe-ß3-Funktionsschicht untersucht. Anschließend werden die $\mathrm{NO}-$ und die $\mathrm{NH}_{3}$-Empfindlichkeiten der Materialien und der gegenseitige Einfluss von $\mathrm{NO}$ und $\mathrm{NH}_{3}$ auf das Sensorsignal charakterisiert. 


\subsection{Fe-Beta}

In Bild 2 sind die Ortskurven eines Fe-ß-Sensors mit einer 20/20 $\mu \mathrm{m}$ IDE-Struktur, aufgenommen bei $500{ }^{\circ} \mathrm{C}$, aufgetragen. Die Kurven zeigen deutliche Halbkreise im hochfrequenten Bereich, die eine RCParallelschaltung repräsentieren, und einen niederfrequenten Elektrodenanteil. Das RC-Parallelglied setzt sich aus der Kapazität der Elektrode und der Zeolithschicht und dem Widerstand der Zeolithschicht zusammen. Im linken Teil ist der Einfluss der NO-Konzentration $c_{\mathrm{NO}}$, die stufenweise von 0 auf $150 \mathrm{ppm}$ erhöht wurde, dargestellt. Der Halbkreis ist unbeeinflusst von der NO-Konzentration, nur der Elektrodenanteil ändert sich leicht. Der Einfluss der $\mathrm{NH}_{3}$-Konzentration dagegen ist deutlich erkennbar (Bild 2, rechts), der Halbkreis wird mit steigender $\mathrm{NH}_{3}$-Konzentration kleiner. Dies korreliert mit einer Widerstandsabnahme durch die Adsorption von $\mathrm{NH}_{3}$ an der Fe-ß-Zeolithschicht. Der Sensor reagiert bei $500{ }^{\circ} \mathrm{C}$ folglich sehr deutlich auf die Zugabe von $\mathrm{NH}_{3}$ wobei keine NO-Störempfindlichkeit vorliegt.

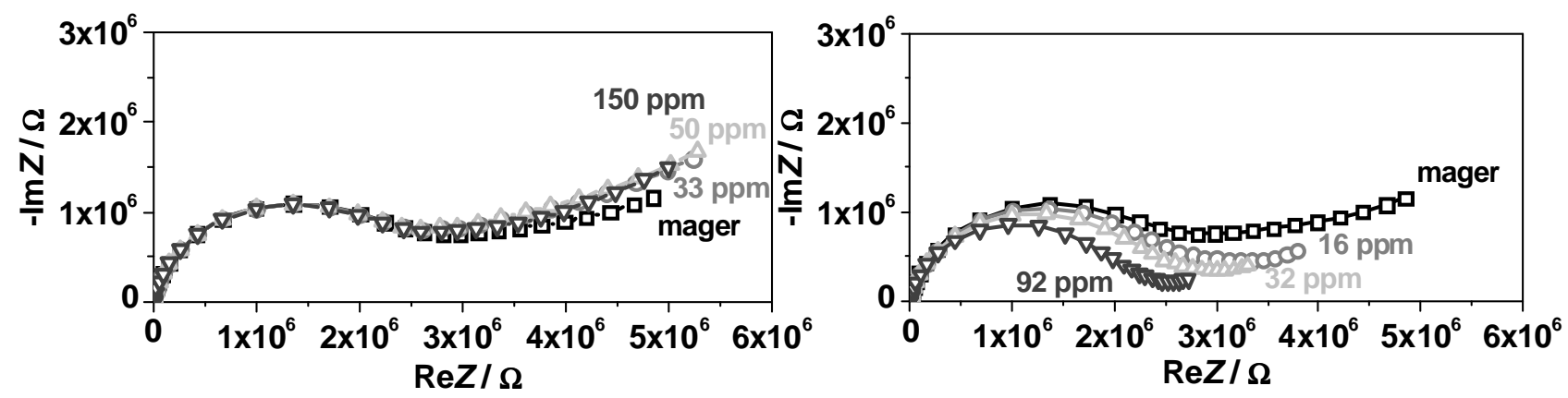

Bild 2 Nyquist-Spektren eines Fe-ß-Zeolithen bei $500{ }^{\circ} \mathrm{C}$ in Abhängigkeit von $c_{\mathrm{NO}}$ (links) und $c_{\mathrm{NH} 3}$ (rechts).

\subsection{Fe-MFI}

Die Ergebnisse des Fe-MFI-Sensors bei $500{ }^{\circ} \mathrm{C}$ sind in Bild 3 dargestellt. Auch mit Fe-MFI als Funktionsschicht werden charakteristische Ortskurven, bestehend aus Halbkreis und Elektrodenanteil, erhalten. Im linken Teil ist die NO-Empfindlichkeit, im rechten das $\mathrm{NH}_{3}$-Sensorsignal dargestellt. Im gemessenen Halbkreis wird durch die Zugabe von NO keine Veränderung hervorgerufen während durch die Zudosierung von $\mathrm{NH}_{3}$ der Durchmesser des Halbkreises kleiner wird. Die Leitfähigkeit nimmt mit steigender $\mathrm{NH}_{3}$-Konzentration zu und der Betrag der komplexen Impedanz $|Z|$ sinkt. Dies ist in Übereinstimmung mit den Ergebnissen der Fe-ß-Sensoren (Bild 2) und mit [2]. Die Adsorption von $\mathrm{NH}_{3}$ führt zu einer mit der $\mathrm{NH}_{3}$-Adsorption proportional ansteigenden Protonenleitfähigkeit, wodurch die Leitfähigkeit erhöht wird. Dagegen wirkt sich die Adsorption von NO am Zeolithen vermutlich nicht auf die Leitfähigkeit aus.

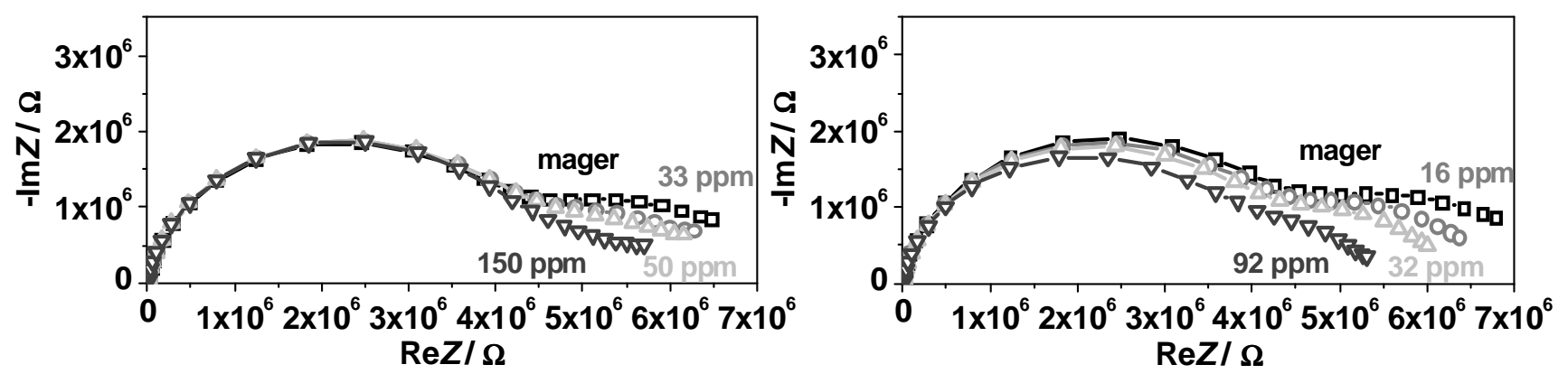

Bild 3 Nyquist-Spektren eines Fe-MFI-Zeolithen bei $500{ }^{\circ} \mathrm{C}$ in Abhängigkeit von $c_{\mathrm{NO}}$ (links) und $c_{\mathrm{NH} 3}$ (rechts).

\subsection{Materialvergleich}

Für eine genauere Analyse der Sensoreigenschaften wurden ein Fe- $\beta$ - und ein Fe-MFI-Sensor mit konstanter Frequenz $\left(f=600 \mathrm{~Hz}\right.$, jeweils im $\mathrm{R} \| \mathrm{C}$-Bereich) bei $450{ }^{\circ} \mathrm{C}$ bzw. $500{ }^{\circ} \mathrm{C}$ vermessen. Der Betrag der komplexen Impedanz $|Z|$ der beiden Sensoren bei Beaufschlagung mit $\mathrm{NO}$ und $\mathrm{NH}_{3}$ in magerem Grundgas ist in Bild 4 gegenübergestellt. 
Beide Sensoren zeigen bei der Beaufschlagung mit NO keinen Sensoreffekt, $|Z|$ ist unabhängig von $c_{\text {No. }}$ Bei $\mathrm{NH}_{3}$-Zugabe zeigt sich eine deutliche Impedanzänderung, $|Z|$ sinkt mit steigender $\mathrm{NH}_{3}$-Konzentration ab. Der Vergleich der Sensorantworten $\left(S=\left|Z_{0}\right|-\left|Z_{\text {Gas }}\right| /\left|Z_{0}\right|\right.$, wobei $\left|Z_{0}\right|$ die komplexe Impedanz im Grundgas und $\left|Z_{\text {Gas }}\right|$ der Wert mit Testgas ist) bei 98 ppm NH 3 liefert $21 \%$ für Fe-MFI und $26 \%$ für Fe- $\beta$. Die NO- und $\mathrm{NO}_{2^{-}}$ Störempfindlichkeiten sind vernachlässigbar, die Sensorantworten liegen bei $0 \%$ (NO) bzw. -2 \% $\left(\mathrm{NO}_{2}\right)$. Die $\mathrm{NO}_{\mathrm{x}}$-Empfindlichkeit ist damit deutlich geringer als für VWT [5].

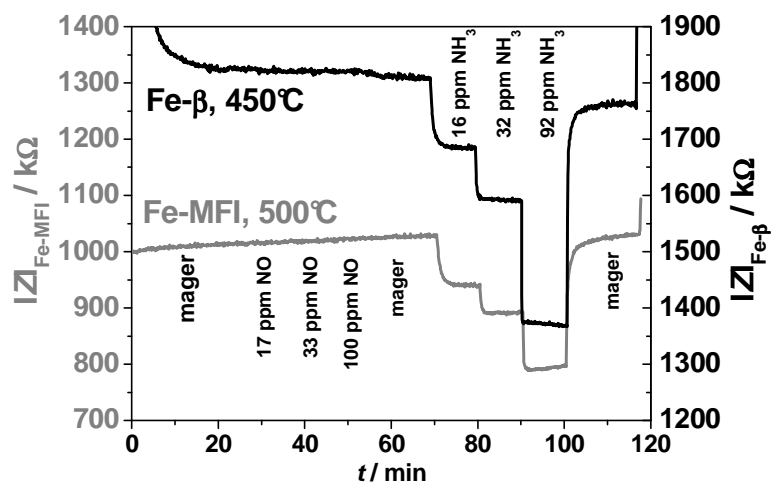

Bild 4 Sensorsignal $|Z|$ auf $\mathrm{NO}$ und $\mathrm{NH}_{3}$ von Fe-MFI- und Fe-ß $-Z$ Zeolith bei $500{ }^{\circ} \mathrm{C}$ bzw. $450{ }^{\circ} \mathrm{C}$.

Der Grund für die starke $\mathrm{NH}_{3}$-Empfindlichkeit liegt vermutlich an der starken $\mathrm{NH}_{3}$-Adsorptionsfähigkeit an den saueren Brönsted-Zentren und der damit verbundenen Erhöhung der Protonenleitfähigkeit. Dies korreliert mit dem in [2] diskutierten Mechanismus für $\mathrm{H}-\beta$-Zeolithe. $\mathrm{NO}$ und $\mathrm{NO}_{2}$ werden dagegen vermutlich weniger stark adsorbiert und die Adsorption trägt im untersuchten Temperaturbereich nicht zu einer Leitfähigkeitsänderung bei.

$\mathrm{Da}$ es sich bei den Materialien um SCR-Katalysatoren handelt, die auch bei Temperaturen um $500{ }^{\circ} \mathrm{C}$ noch SCR-aktiv sind und $\mathrm{NO}_{x}$ mit $\mathrm{NH}_{3}$ konvertieren, ist neben den Sensorantworten auch die gegenseitige Beeinflussung von $\mathrm{NO}$ und $\mathrm{NH}_{3}$ von Bedeutung. Der Einfluss von $\mathrm{NO}$ auf das $\mathrm{NH}_{3}$-Sensorsignal ist in Bild 5 für beide Sensoren, gemessen bei $450{ }^{\circ} \mathrm{C}$ und $600 \mathrm{~Hz}$, gezeigt. Liegt kein $\mathrm{NH}_{3}$ im Gas vor, so ist $|Z|$ unabhängig von der NO-Konzentration. Werden $20 \mathrm{ppm} \mathrm{NH}$ zugegeben, sinkt $|Z| \mathrm{ab}$, die Sensorantwort beträgt $11 \%$. Durch die Zugabe von NO sinkt die Sensorantwort für Fe-MFI auf 4,3\% (65 ppm NO) bzw. 2,1 \% (150 ppm NO) ab. Der Fe-ß3-Sensor weist einen etwa gleichgroßen NO-Einfluss auf. Mit steigender $\mathrm{NH}_{3}$-Konzentration nimmt der NO-Einfluss leicht ab.
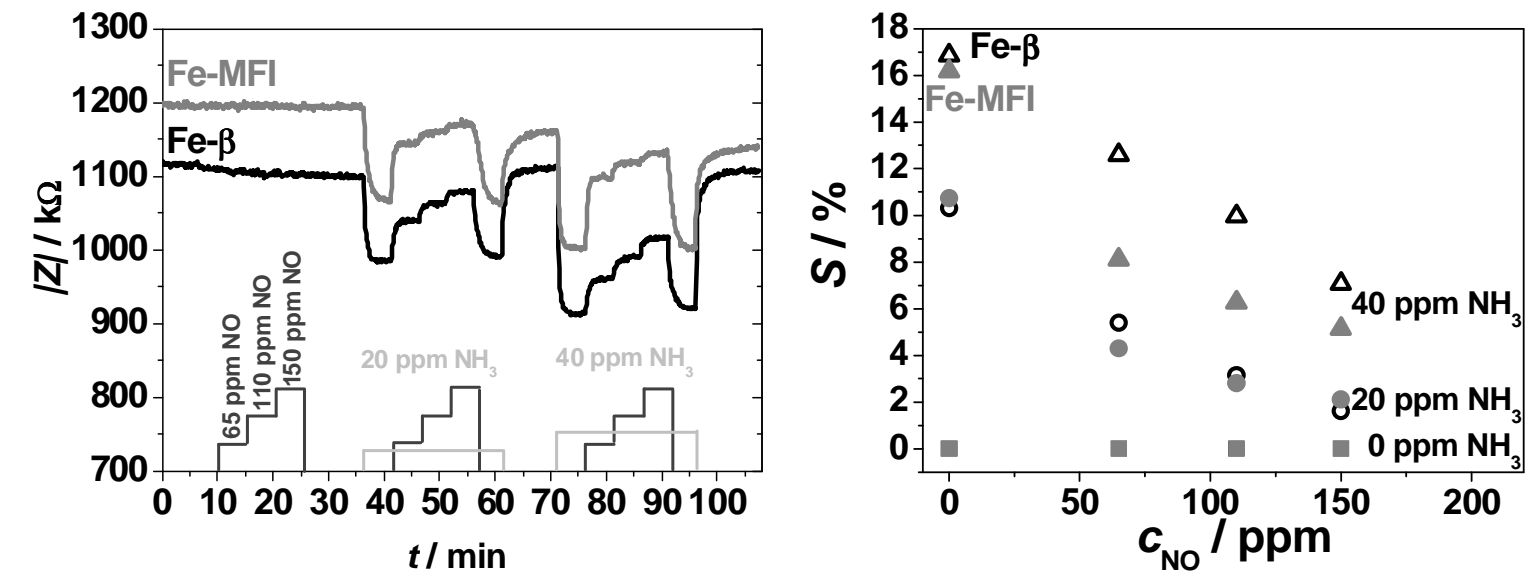

Bild 5 NO-Einfluss auf das $\mathrm{NH}_{3}$-Sensorsignal $|Z|$ (links) und auf die Sensorantwort $S$ (rechts) für einen Fe-MFI- und FeB-Zeolith bei $450{ }^{\circ} \mathrm{C}$.

Das $\mathrm{NH}_{3}$-Signal wird bei beiden Materialien deutlich von der NO-Konzentration beeinträchtigt, die Sensorantwort auf $\mathrm{NH}_{3}$ nimmt ab. Dies ist auf die SCR-Aktivität der Zeolithe zurückzuführen, denn das adsorbierte $\mathrm{NH}_{3}$, das für den Sensoreffekt verantwortlich ist, wird mit gasförmigen NO umgesetzt und kann somit nicht mehr zur Änderung der Leitfähigkeit beitragen. Die Sensorantwort nimmt folglich ab. 


\section{Fe-SAPO-5 als impedimetrischer Gassensor}

Fe-SAPO sind nach [4] hydrothermal stabilere Alternativen zu Fe-MFI-basierten SCR-Katalysatoren. Erste Ergebnisse der impedimetrischen Charakterisierung von Fe-SAPO-5 mit einem 15/15 $\mu \mathrm{m}$ IDE sind in Bild 6 dargestellt. Zum mageren Grundgas wurden bei $400{ }^{\circ} \mathrm{C}$ (Bild 6, links) und $500{ }^{\circ} \mathrm{C}$ (Bild 6, rechts) $475 \mathrm{ppm}$

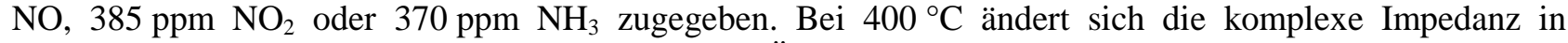
Abhängigkeit vom Testgas sehr deutlich. Die größte Änderung wird durch $\mathrm{NO}_{2}$ hervorgerufen, die Impedanz nimmt deutlich ab. Auch durch $\mathrm{NH}_{3}$ wird die Impedanz verringert, während NO keinen Einfluss hat. Bei $500{ }^{\circ} \mathrm{C}$ zeigt sich keine Änderung im Halbkreis, er ist unabhängig von der Gaszusammensetzung. Nur im niederfrequenten Bereich ist bei $\mathrm{NH}_{3}$-Zugabe eine Änderung erkennbar. Der Effekt bei $400{ }^{\circ} \mathrm{C}$ wird der Adsorption von $\mathrm{NO}_{2}$ bzw. $\mathrm{NH}_{3}$ am Fe-SAPO-5 zugeschrieben und einer dadurch veränderten Leitfähigkeit. Für die Änderung im niederfrequenten Anteil bei $500{ }^{\circ} \mathrm{C}$ könnten Elektrodeneffekte verantwortlich sein, die jedoch noch detaillierter untersucht werden müssen.
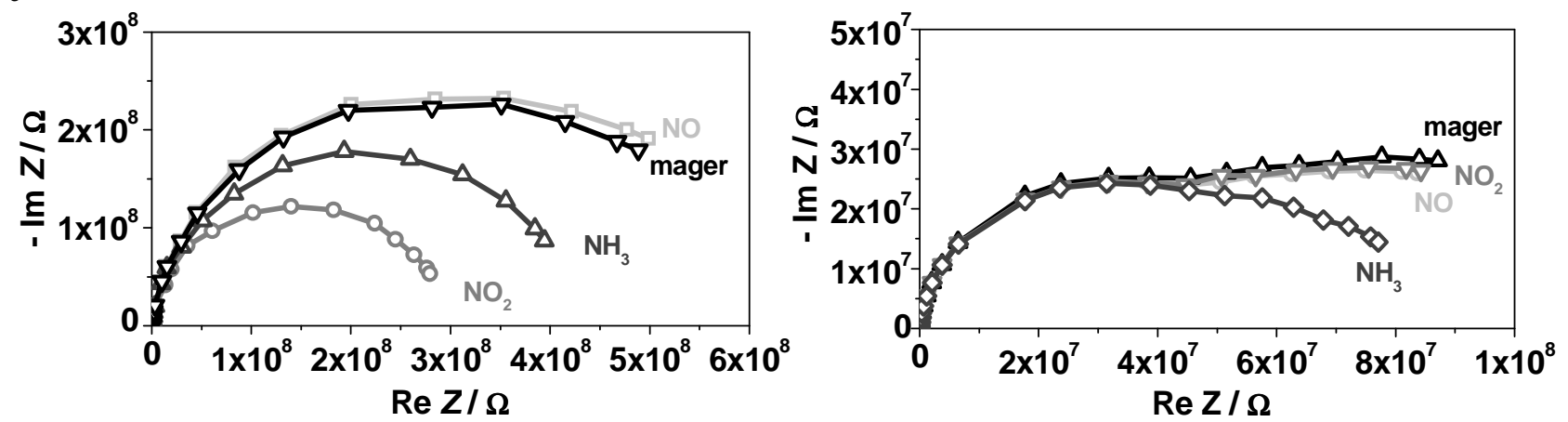

Bild 6 Ortskurven eines Fe-SAPO-5-Sensors bei $400{ }^{\circ} \mathrm{C}$ (links) und $500{ }^{\circ} \mathrm{C}$ (rechts) in Abhängigkeit von $\mathrm{NO} \mathrm{NO}_{2}$ und $\mathrm{NH}_{3}$ in magerem Grundgas, IDE $15 / 15 \mu \mathrm{m}$.

\section{Zusammenfassung}

Der Vergleich der komplexen Impedanzen der untersuchten Materialien zeigt, dass der Fe-SAPO-5 die geringste Leitfähigkeit besitzt, während Fe-MFI und Fe-ß gleichgroße Leitfähigkeiten aufweisen. Die impedimetrischen Sensoren auf Fe-Zeolith-Basis reagieren auf $\mathrm{NH}_{3}$ ausgesprochen empfindlich. Der Betrag der komplexen Impedanz $|Z|$ nimmt mit steigender $\mathrm{NH}_{3}$-Konzentration ab, d.h. die Leitfähigkeit des Zeolithen wird durch die Adsorption von $\mathrm{NH}_{3}$ erhöht. Die $\mathrm{NO}_{\mathrm{x}}$-Störempfindlichkeit dagegen ist vernachlässigbar. Jedoch wird das $\mathrm{NH}_{3}$-Signal deutlich von der $\mathrm{NO}_{\mathrm{x}}$-Hintergrundkonzentration beeinflusst, was auf die SCR-Aktivität der Fe-Zeolithe zurückzuführen ist. Erste Ergebnisse zum Einsatz von hydrothermal stabileren Fe-SAPO-5 als Sensorschicht zeigen bei $400{ }^{\circ} \mathrm{C}$, nicht aber bei $500^{\circ} \mathrm{C}$, eine $\mathrm{NO}_{2^{-}}$ und $\mathrm{NH}_{3}$-Abhängigkeit. Die verschiedenen SCR-Katalysatormaterialien eignen sich als funktionelle Sensorschichten, jedoch sind weitere Untersuchungen zum gassensitiven Verhalten und der Stabilität notwendig.

\section{Literatur}

[1] K. Alberti, F. Fetting, Zeolites as sensitive materials for dielectric gas sensors, Sensors and Actuators B 21 (1994) 39-50.

[2] U. Simon, U. Flesch, W. Maunz, R. Müller, C. Plog, The effect of $\mathrm{NH}_{3}$ on the ionic conductivity of dehydrated zeolites Na-beta and H-beta, Microporous Mesoporous Materials 21 (1998) 111-116.

[3] O. Kröcher, Martin Elsener, Combination of $\mathrm{V}_{2} \mathrm{O}_{5} / \mathrm{WO}_{3}-\mathrm{TiO}_{2}, \mathrm{Fe}-\mathrm{ZSM} 5$, and Cu-ZSM5 Catalysts for the Selective Catalytic Reduction of Nitric Oxide with Ammonia, Industrial and Engineering Chemistry Research 47 (2008) 8588-8593.

[4] M. Schwidder, P. G. W. A. Kompio, W. Grünert , F. Scheffler, Iron-modified SAPOs as Hydrothermally Stable Catalysts for DeNOx Applications, Proceedings 22. Deutsche Zeolith-Tagung München (2010) 158-159.

[5] D. Schönauer, I. Sichert, R. Moos, Vanadia doped tungsten-titania SCR catalysts as functional materials for exhaust gas sensor applications, Sensors and Actuators B 155 (2011) 199-205.

[6] N. Izu, G. Hagen, D. Schönauer, U. Röder-Roith, R. Moos, Application of $\mathrm{V}_{2} \mathrm{O}_{5} / \mathrm{WO}_{3} / \mathrm{TiO}_{2}$ for Resistive-Type $\mathrm{SO}_{2}$ Sensors, Sensors 11 (2011) 2982-2991. 